\title{
The Short-Term Effect of Active Skeletonized Sutural Distractor Appliance on Temporomandibular Joint Morphology of Class III Malocclusion Subjects
}

Mohamed Zahoor Ul Huqh ${ }^{1}$ Rozita Hassan ${ }^{1}$ Wael Mohamed ${ }^{1}$ Roselinda Abdul Rahman ${ }^{2}$

Asilah Yusof $^{3}$ Ida Bagus Narmada ${ }^{4}$ Wan Muhamad Amir W Ahmad ${ }^{5}$

\begin{abstract}
Address for correspondence Rozita Hassan, BDS, MOrth (Malaya), MOrth (Edinburgh), Orthodontic Unit, School of Dental Sciences, Universiti Sains Malaysia, Kelantan 16150, Malaysia (e-mail: rozitakb@usm.my).

Roselinda Abdul Rahman, BDS (Malaya), MClinDent-Oral Surgery (Malaya), Department of Oral and Maxillofacial Surgery, School of Dental Sciences, Universiti Sains Malaysia Health Campus, Kelantan 16150, Malaysia (e-mail: roselinda@usm.my).
\end{abstract}

\section{Abstract}

Keywords

- class III malocclusion

- temporomandibular joint morphology

- orthodontic treatment

- fixed appliance

- cone-beam computed tomography
Objectives The purpose of this study was to evaluate the short-term effect of active skeletonized sutural distractor (ASSD) appliance on temporomandibular joint morphology of class III malocclusion subjects.

Materials and Methods This was a prospective interventional study. Cone-beam computerized tomography ( $\mathrm{CBCT}$ ) images of 22 patients were taken before and after treatment by using Planmeca Promax 3D CBCT machine version 2.9.2 (Planmeca OY Helsinki, Finland). The condylar width, height, length, roof of glenoid fossa thickness, and all joint spaces were measured. The condylar position was determined based on Pullinger and Hollander formula. The condylar shape was determined as per Kinzinger et al. The condylar volume was calculated by using Mimics software (Materialize, Belgium).

Statistical Analysis Data analysis was performed by using SPSS software version 24. Wilcoxon paired signed-rank test was used to compare the difference in temporomandibular joint morphology and condylar volume between pre- and post-treatment measurements. Chi-square test was used to compare the condylar position and shape.

Results The superior ( $p=0.000$ on the right side, $p=0.005$ on the left side) and posterior joint spaces ( $p=0.000$ on both sides) were decreased after the treatment, respectively. The condyles were rotated upward and backward, thereby increasing the anterior joint spaces ( $p=0.000$ on both sides) after the treatment. The condylar volume increases post treatment, however, no significant differences were observed ( $p=0.903$ on the right side, $p=0.062$ on the left side).

Conclusion The significant changes were observed in joint spaces. The condyles were more anteriorly placed before treatment. Condylar position and shape alter in response to ASSD treatment. The condylar volume did not show any significant change. published online

February 23, 2021
DoI https://doi.org/

10.1055/s-0040-1722483 ISSN 1305-7456.

\footnotetext{
(c) 2021. European Journal of Dentistry.

This is an open access article published by Thieme under the terms of the Creative Commons Attribution-NonDerivative-NonCommercial-License, permitting copying and reproduction so long as the original work is given appropriate credit. Contents may not be used for commercial purposes, or adapted, remixed, transformed or built upon. (https://creativecommons.org/licenses/by-nc-nd/4.0/)

Thieme Medical and Scientific Publishers Pvt. Ltd., A-12, 2nd Floor, Sector 2, Noida-201301 UP, India
} 


\section{Introduction}

The global prevalence rate of class III malocclusion ranged between 0 and 26.7\%. The South-East Asians showed the highest prevalence rate of approximately 12.58 to $26.7 \% .^{1,2}$ Overall, $14.7 \%$ of Malaysian has class III malocclusion. ${ }^{3}$ While Middle Eastern and the Japanese nations had a mean prevalence of 10.18 and 2.3 to $14 \%$, respectively. ${ }^{4-6} \mathrm{~A}$ low prevalence rate of the malocclusion was observed among Americans (5\%), European nations (4.88\%), ${ }^{7.8}$ and African nations (4.59\%).,10 The Indian population had the lowest prevalence rate ranged between 0 to $4.76 \%{ }^{11,12}$

The class III malocclusion might be presented with mandibular prognathism, maxillary retrognathism, protrusive mandibular dentition or retrusive maxillary dentition, or a combination of these components. In the Asian population, $60 \%$ of the class III malocclusion patients aged between 6 to 14 years old exhibited midface deficiency. ${ }^{13}$ Most Korean patients had a normal maxilla and overdeveloped mandible (47.7\%), with fewer patients having an underdeveloped maxilla and overdeveloped mandible (13.5\%). Among Malaysians, class III malocclusion subjects age ranged between 12 to 25 years old had protruded mandible with short cranial base. ${ }^{3}$

A new device has been fabricated by a group of researchers in the orthodontic unit, School of Dental Sciences, Universiti Sains Malaysia (USM) known as active skeletonized sutural distractor (ASSD). It is indicated for adolescent patients with moderate to severe class III malocclusion and indicated for orthognathic surgery is the treatment of choice. This appliance is used to overcome the limitations of orthognathic surgery. The ASSD is cost-effective, reduces patient discomfort, and corrects skeletal class III malocclusion within a short duration of 6 months when compared with Facemask therapy and other treatment methods. The appliances such as Delaire face mask, Jasper Jumper, Reverse twin block, and multiloop edgewise arch wire (MEAW) with modified class III elastics were used in previous literature, but no signs and symptoms of temporomandibular joint (TMJ) have been reported.

Patient with moderate to severe class III malocclusion had to bear the deformity until an appropriate age for orthognathic surgery. Apart from that, the surgery is expensive and invasive with a relatively extended recovery period from 6 to 12 weeks. It is also associated with discomfort, soft diet intake, facial swelling, transient paresthesia, and sometimes permanent loss of neurosensation. ${ }^{14}$ The intraoperative complications include insufficient osteotomy, bleeding due to injuries of blood vessels, nerve damage, dental, and soft tissue injuries among the patients..$^{15}$

The mandibular condyle is considered as the main feature of the TMJ structure. It responds to continuous stimuli from early childhood to adulthood and undergoes remodeling process, which is considered as the center of growth in the mandible where its shape and volume could be associated with upper and lower bases. ${ }^{16-18}$

TMJ is a synovial joint. It is one of the most complex joints in the body. A sound understanding of the TMJ morphology is essential to the study of function and dysfunction in the masticatory system. The variation in morphology may cause jaw deformity, facial asymmetry, joint pain, disc displacement, and perforation. Latest advances have been encouraged the use of three-dimensional computed tomography (CT) as an accurate diagnostic imaging technique for maxillofacial morphology. However, the use of CT in dentistry is restricted due to high exposure of radiation dose and low resolution of the images in an axial direction. Cone-beam computerized tomography (CBCT) is advantageous for dental and maxillofacial imaging particularly in the axial direction due to its high-resolution images ${ }^{19}$ and less scan time with a radiation dose of 8 to 333 microsieverts $(\mu \mathrm{Sv}) .^{20}$

CBCT is economical, requires less radiation exposure when compared with medical CT scans, image acquisition is easy, and displays interactive modes like multiplanar reconstruction in which images can be displayed in axial, coronal, and sagittal views. As CBCT provides three-dimensional images, TMJ can be viewed in multiple planes. This study aimed to evaluate the short-term effect of ASSD on TMJ morphology of class III malocclusion subjects.

\section{Materials and Methods}

This was a prospective interventional study involving 22 subjects among which 9 males and 13 females with the mean age of $15 \pm 1.4$ years and the age group between 12 to 17 years were treated consecutively with ASSD appliance. Subjects with the following criteria were included:

- Moderate to severe class III malocclusion with reverse overjet of $2 \mathrm{~mm}$ or more

- Maxillary retrognathism with or without mandibular prognathism

- Undergoing treatment with ASSD

- Good quality of CBCT images

Subjects with (1) systemic disease, TMDs and congenital anomalies such as cleft lip and cleft palate, (2) history of orthodontic treatment, (3) pseudo class III malocclusion patients, (4) history of any tenderness in the joint, clicking sound, and deviation of the jaw were excluded from the study.

Ethical approval was obtained from the Human Research Ethics committee of Universiti Sains Malaysia (approval number USM/JEPeM/15120548). The study procedure was explained to the patients. Written informed consent was obtained from both patients and their parents or legally acceptable representative for the documentation and public presentation of their data.

\section{Components of Active Skeletonized Sutural Distractor Appliance}

The ASSD consists of two miniscrew implants in the anterior palate, an expansion screw (Miniexpander, Ortho-Care UK Ltd) is incorporated into a metallic framework, which is attached to the molar bands as shown in (-Fig. 1). The appliance on the lower arch comprised stainless steel archwire of size $2 \mathrm{~mm}$ that is attached to molar bands and between canines and first premolars a wire loop is soldered to the archwire to facilitate elastic attachment from buccal tubes of upper molars to the wire loop as shown in (-Fig. 2). 


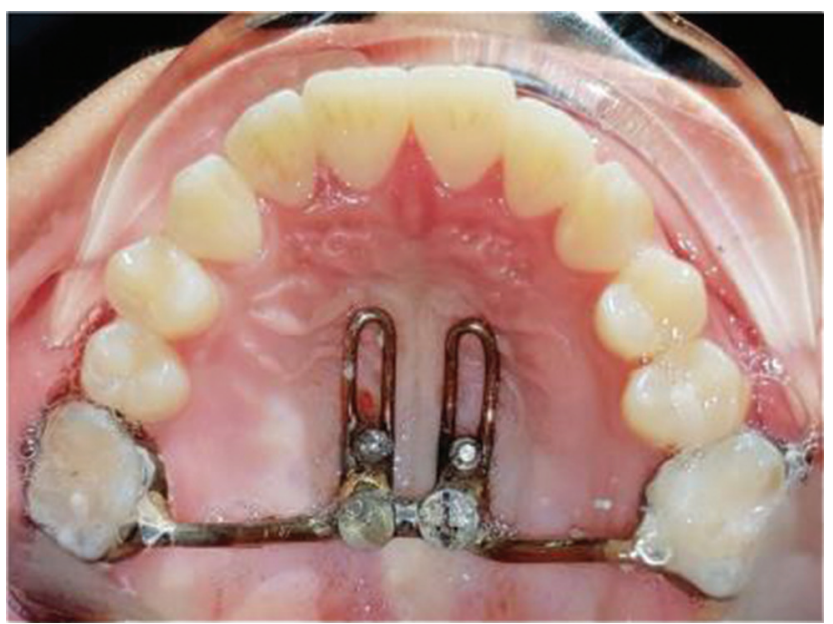

Fig. 1 Represents active skeletonized sutural distractor appliance design on upper arch.

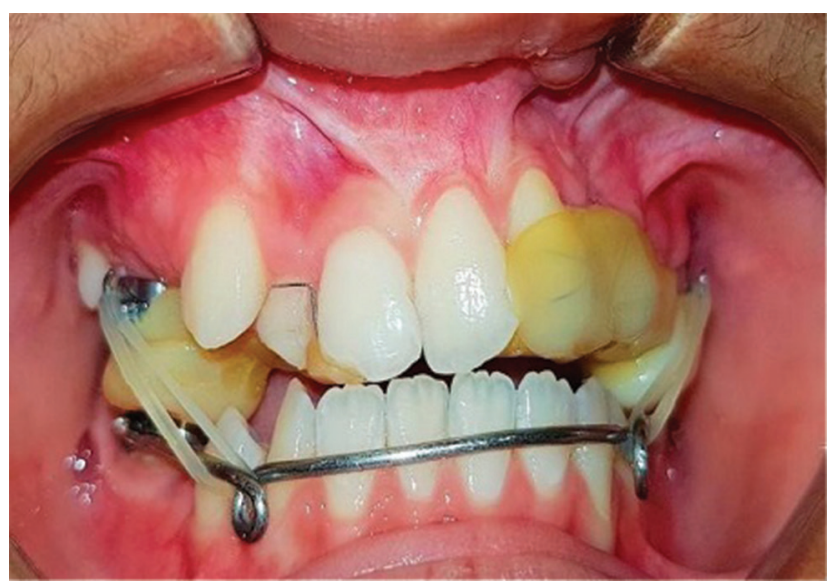

Fig. 2 Represents active skeletonized sutural distractor appliance design on lower arch.

\section{Clinical Protocol}

The clinical protocol for ASSD is described in (-Table $\mathbf{1}$ ). After the insertion of the ASSD appliance, a force of $500 \mathrm{~g}$ per side was applied by using heavy class III elastics. The direction of the force was approximately 30 degrees downward to the line of occlusion. During the active treatment phase, the patients were instructed to wear the class III elastics full time and perform alternate rapid maxillary expansion and constriction (Alt-RAMEC) by turning the expansion screw 8 turns every 8 hours (morning, afternoon, and evening) to expand the maxilla. The patients were advised to reverse the number of turns to constrict the maxilla for the next 3 days, and repeated for approximately 12 weeks or until the positive overjet and desired expansion of 6 to $8 \mathrm{~mm}$ was achieved. During the retention phase, patients were instructed to wear the clear retainer with class III elastics applying $250 \mathrm{gm}$ force per side for 6 months.

\section{Scanning Procedure}

Patients were scanned by using standardized TMJ view (Field of view of $8 \times 8 \mathrm{~cm}$, voxel size $0.25 \mathrm{~mm}, 90 \mathrm{KVp}$ tube voltage,
Table 1 Clinical protocol for active skeletonized sutural distractor appliance

\begin{tabular}{|c|c|}
\hline \multicolumn{2}{|l|}{ Pretreatment data collection } \\
\hline Clinical examination sheet & \\
\hline \multicolumn{2}{|l|}{ lateral cephalometric } \\
\hline \multicolumn{2}{|l|}{ Panoramic X-ray } \\
\hline \multicolumn{2}{|l|}{ Study model } \\
\hline \multicolumn{2}{|l|}{ Facial photos } \\
\hline \multicolumn{2}{|l|}{ Consent form } \\
\hline \multicolumn{2}{|l|}{ OHIP 14 questionnaire } \\
\hline \multicolumn{2}{|l|}{ Working model } \\
\hline \multicolumn{2}{|l|}{ Delivery of the ASSD appliance } \\
\hline \multicolumn{2}{|l|}{$\begin{array}{l}\text { The patient instructed to wear class III elastic full } \\
\text { time }\end{array}$} \\
\hline \multicolumn{2}{|l|}{$\begin{array}{l}\text { The patient instructed to perform Alt-RAMEC by } \\
\text { turning the super screw } 8 \text { turns every } 8 \text { hours } \\
\text { (morning, afternoon, evening) to expand the } \\
\text { maxilla for } 3 \text { days }\end{array}$} \\
\hline \multicolumn{2}{|l|}{ Patient follow-up } \\
\hline $\begin{array}{l}\text { On the 4th day of delivery, an upper impression } \\
\text { will be taken and the mobility of the miniscrews } \\
\text { will be assessed and the patient instructed to } \\
\text { reverse the turn to constrict the maxilla for the } \\
\text { next } 3 \text { days then reverse the turn and repeat the } \\
\text { Alt-RAMEC until the end of the treatment period }\end{array}$ & \\
\hline \multicolumn{2}{|l|}{$\begin{array}{l}\text { Then patient follow-up will be performed every } \\
2 \text { weeks to assess the correction of the maloc- } \\
\text { clusion and mobility of the miniscrews until the } \\
\text { end of the treatment period }\end{array}$} \\
\hline \multicolumn{2}{|l|}{ Post-treatment data collection } \\
\hline \multicolumn{2}{|l|}{ Allocation of the treatment period } \\
\hline \multicolumn{2}{|l|}{ Mobility test } \\
\hline \multicolumn{2}{|l|}{ Lateral cephalometric } \\
\hline \multicolumn{2}{|l|}{$\begin{array}{l}\text { OHIP } 14 \text { questionnaire after } 1 \text { month from } \\
\text { finishing treatment }\end{array}$} \\
\hline \multicolumn{2}{|l|}{ Retention period } \\
\hline $\begin{array}{l}\text { Patient instructed to wear the clear retainer } \\
\text { with class III elastics applying } 250 \text { gm force for } \\
6 \text { months }\end{array}$ & \\
\hline
\end{tabular}

Abbreviations: Alt-RAMEC, alternate rapid maxillary expansion and constriction; ASSD, active skeletonized sutural distractor; OHIP, oral health impact profile.

and $10 \mathrm{~mA}$ tube current for 10 to 12 seconds with standing posture) before and after 6 months of ASSD treatment. CBCT images were taken by using Planmeca Promax 3D CBCT machine (Planmeca OY Helsinki, Finland) to assess the condylar parameters and position within the glenoid fossa of TMJ.

The head was positioned with Frankfort horizontal plane parallel to the floor. Both pre-and postoperative CBCT data were transferred to the DICOM file format. Prior to measurement, training was obtained from the craniofacial imaging expert. Calibration was done by using an electronic scale measurement tool in Planmeca Romexis imaging 
software version 2.9.2. The reference points are described in (-Table 2). Linear measurements were performed on the sagittal slice of CBCT with the help of both axial and coronal slices as shown in (-Table $\mathbf{3})$. The measurements were done based on the methodology described by Al-koshab et al. ${ }^{21}$

The following measurement has been described below:

- The condylar length was measured on a mid-sagittal slice from posterior condylar point ( $\mathrm{PCo}$ ) to the anterior condylar point (ACo) as illustrated in ( - Fig. $\mathbf{3}$ ).

- The condylar width was measured on a mid-coronal plane between the medial (MCo) and lateral (LCo) condylar point as illustrated in ( - Fig. 4).

- Condylar height was measured on a mid-sagittal plane between a perpendicular line from superior condylar point (SCo) to a tangential line drawn from the most inferior point of the sigmoid notch (InfSig) as illustrated in ( - Fig. 5).

- The roof of glenoid fossa (RGF) was measured in a mid-sagittal plane at the location of superior fossa (SF) as illustrated in (-Fig. 6).

- Joint space: THL was drawn which is tangential to the most superior point of the RGF and a line tangent to SCo is also drawn. Then the two other imaginary lines were drawn connecting the SF to ACo and SF to PCo as illustrated in ( - Fig. 7A)

- Superior joint space (SJS) was measured from the superior fossa SF to SCo, anterior joint space (AJS) was

Table 2 The reference points used for measurement ${ }^{21}$

\begin{tabular}{|l|l|l|}
\hline $\begin{array}{l}\text { Superior } \\
\text { condylar } \\
\text { point }\end{array}$ & SCo & $\begin{array}{l}\text { The most superior point on the } \\
\text { mandibular condyle }\end{array}$ \\
\hline $\begin{array}{l}\text { Posterior } \\
\text { condylar point }\end{array}$ & PCo & $\begin{array}{l}\text { The most posterior point on the } \\
\text { mandibular condyle }\end{array}$ \\
\hline $\begin{array}{l}\text { Anterior } \\
\text { condylar point }\end{array}$ & ACo & $\begin{array}{l}\text { The most anterior point on the } \\
\text { mandibular condyle }\end{array}$ \\
\hline $\begin{array}{l}\text { Medial } \\
\text { condylar point } \\
\text { and lateral } \\
\text { condylar point }\end{array}$ & $\begin{array}{l}\text { MCo } \\
\text { LCo }\end{array}$ & $\begin{array}{l}\text { The medial and lateral poles of the } \\
\text { condyles are the points at which } \\
\text { articular disc attaches to the discal } \\
\text { ligaments }\end{array}$ \\
\hline $\begin{array}{l}\text { Superior fossa } \\
\text { SF }\end{array}$ & $\begin{array}{l}\text { The most superior point on the glenoid } \\
\text { fossa }\end{array}$ \\
\hline $\begin{array}{l}\text { Inferior } \\
\text { sigmoid notch }\end{array}$ & InfSig & $\begin{array}{l}\text { The most inferior point of the sigmoid } \\
\text { notch }\end{array}$ \\
\hline
\end{tabular}

Table 3 Linear measurements ${ }^{21}$

\begin{tabular}{|l|l|l|}
\hline $\begin{array}{l}\text { True horizontal } \\
\text { line }\end{array}$ & THL & $\begin{array}{l}\text { A reference plane which is tangential to } \\
\text { the most superior point of the roof of } \\
\text { glenoid fossa }\end{array}$ \\
\hline $\begin{array}{l}\text { Roof of glenoid } \\
\text { fossa }\end{array}$ & RGF & $\begin{array}{l}\text { A line drawn from the lowermost point } \\
\text { of glenoid fossa to the SF }\end{array}$ \\
\hline $\begin{array}{l}\text { Superior joint } \\
\text { space }\end{array}$ & SJS & A line drawn from the SCo to the SF \\
\hline $\begin{array}{l}\text { Anterior joint } \\
\text { space }\end{array}$ & AJS & $\begin{array}{l}\text { A line drawn from the ACo to the point } \\
\text { at which the imaginary line contacting } \\
\text { the anterior wall of the glenoid fossa }\end{array}$ \\
\hline $\begin{array}{l}\text { Posterior joint } \\
\text { space }\end{array}$ & PJS & $\begin{array}{l}\text { A line drawn from the PCo to the point } \\
\text { at which the imaginary line contacting } \\
\text { the posterior wall of the glenoid fossa }\end{array}$ \\
\hline
\end{tabular}

measured from ACo to the point on the anterior wall of the glenoid fossa where the tangential line met the anterior wall of the glenoid fossa and posterior joint space (PJS) was measured from PCo to the point on the posterior wall of glenoid fossa where the tangential line met the posterior wall of the glenoid fossa as illustrated in ( - Fig. 7B).
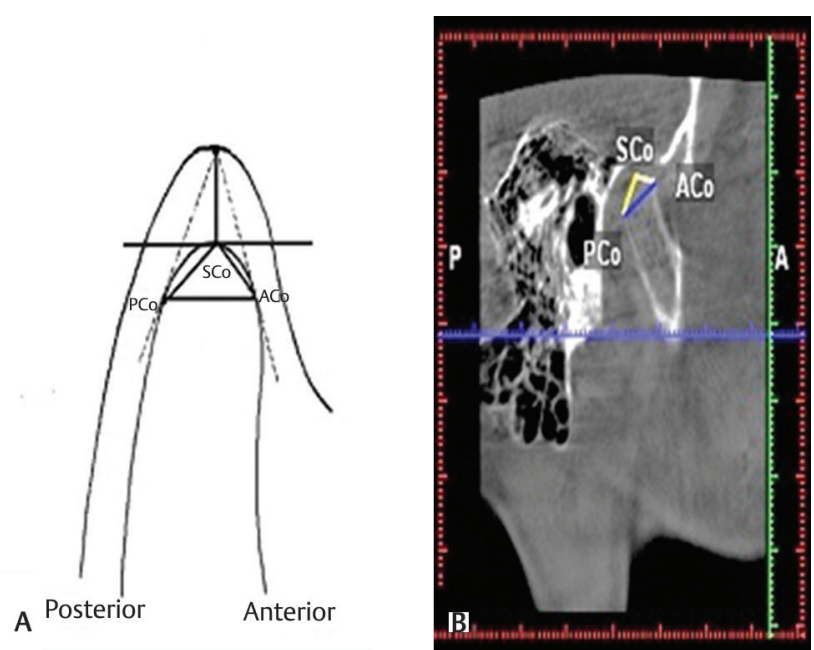

Fig. 3 (A) Schematic diagram showing condylar length measurement and (B) mid-sagittal view showing condylar length measurement.

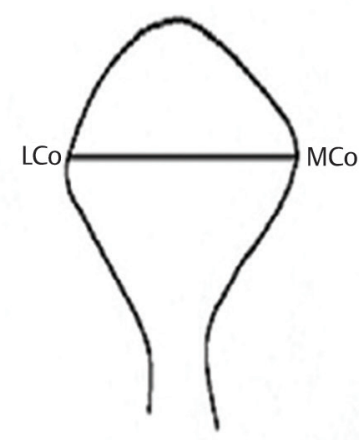

A

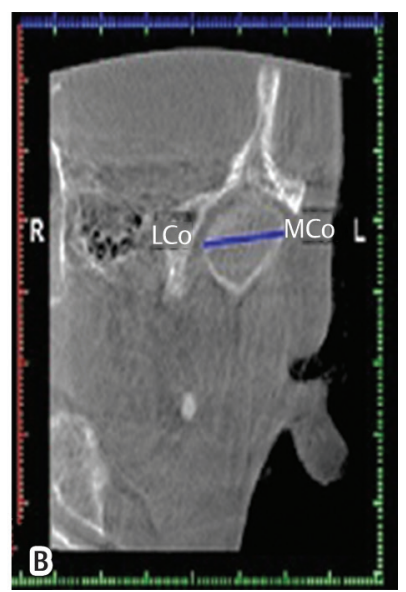

Fig. 4 (A) Schematic diagram showing condylar width measurement and (B) mid-coronal view showing condylar width measurement.

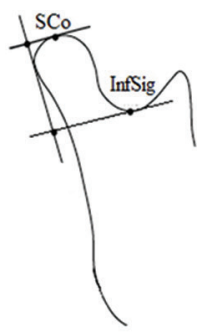

A

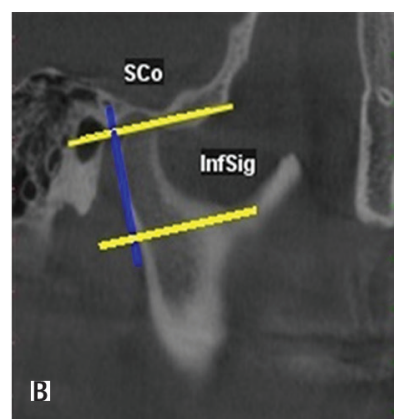

Fig. 5 (A) Schematic diagram showing condylar height measurement and (B) mid-sagittal view showing condylar height measurement. 


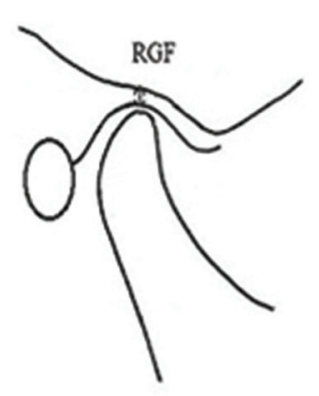

A

Fig. 6 (A) Schematic diagram showing roof of glenoid fossa measurement and (B) mid-sagittal view showing roof of glenoid fossa measurement.
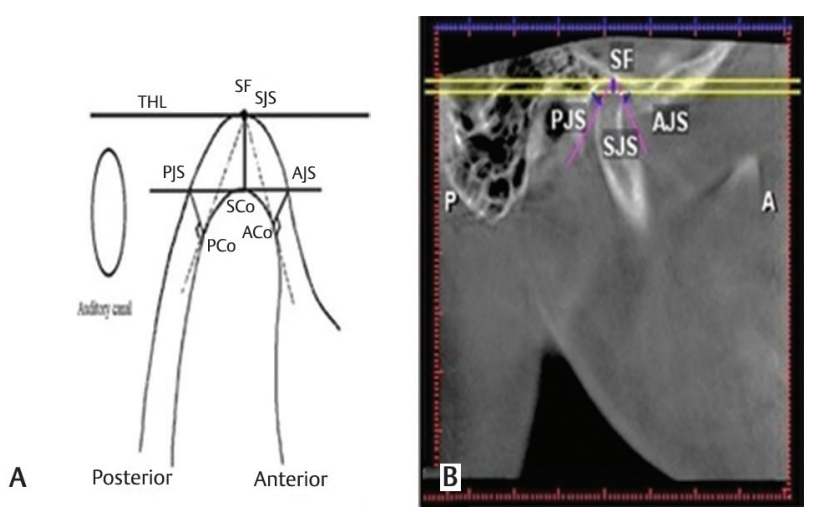

Fig. 7 (A) Schematic diagram showing joint space measurement and (B) mid-sagittal view showing joint space (anterior joint space, posterior joint space, and superior joint space) measurement.

\section{Condylar Position}

The position of the condyle within the glenoid fossa can be determined via its linear ratio. This was calculated using the following formula given by Pullinger and Hollander. ${ }^{22}$

Linear ratio $=(\mathrm{P}-\mathrm{A}) /(\mathrm{P}+\mathrm{A}) \times 100$, where $\mathrm{P}=\mathrm{PJS}, \mathrm{A}=\mathrm{AJS}$

The following values were used to describe the condylar position:

- -12 to $+12=$ condyle is in concentric position

- $<-12=$ condyle is in posterior position

- $>12=$ condyle is in anterior position

\section{Condylar Shape}

As per Kinzinger et al, ${ }^{23}$ shape of the condyle was determined as round, convex, and angulated which can be viewed in mid coronal view as shown in (-Fig. 8).

\section{Condylar Volume}

CBCT datasets were imported into Mimics Software (Materialize, NV, Belgium). The imported images were segmented by using mimics tool to edit the mask and build a threshold area through which isolation of only the condylar part was done. The remaining slices of the images were erased in all the views except the mandibular condyle. After the isolation, three-dimensional images were
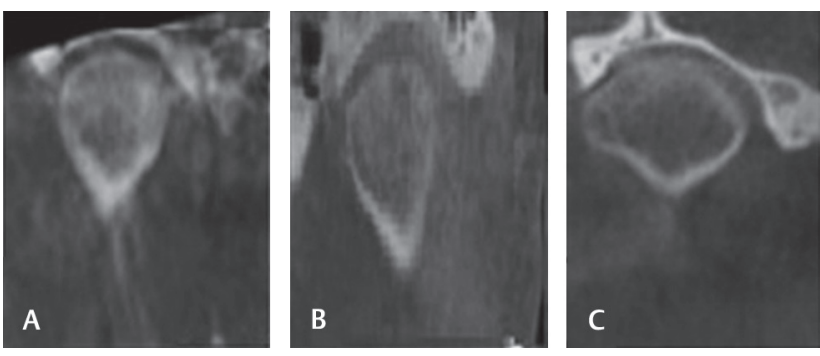

Fig. 8 Shape of the condyle. (A) Round, (B) angulated, and (C) convex.

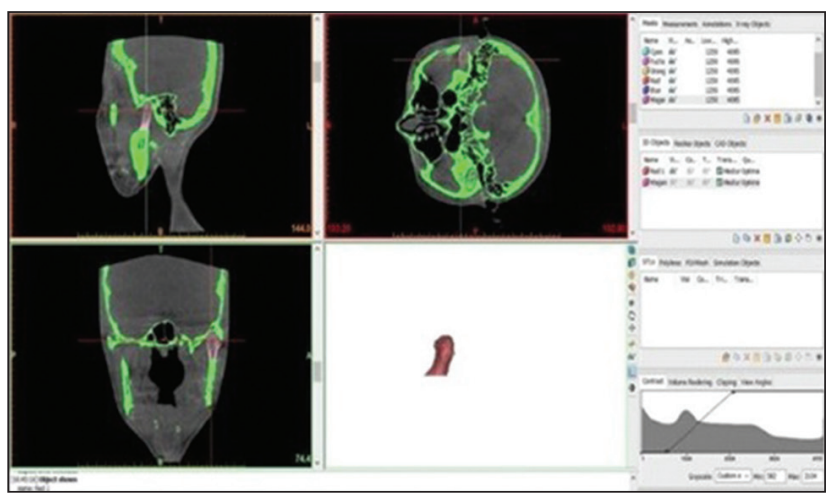

Fig. 9 Determination of condylar volume using Mimics software (Materialize Belgium) with automated function.

reconstructed by calculating the volume of each condyle with automated function as shown in ( - Fig. 9).

\section{Statistical Analysis}

SPSS software version 24 (IBM SPSS Statistics; Armonk, New York, United States) was used to perform the data analysis. Descriptive statistics such as median and interquartile range (IQR) has been calculated. The $p<0.05$ was considered as statistically significant. Wilcoxon paired signed-rank test was used to compare the difference in TMJ morphology and condylar volume between pre- and post-treatment measurements. Chi-square test for goodness of fit was used to compare the condylar position and shape between pre- and post-treatment measurements.

\section{Measurement Reliability}

Overall, $20 \%$ of the sample was randomly selected to assess the reliability and remeasured by two examiners. Both intra- and interexaminer reliability measurement were done within 2 weeks interval. The difference in measurement was calculated by using intraclass correlation coefficient at a $95 \%$ confidence interval $(\mathrm{CI})$. The variables displayed the values in the range between 0.8 and 0.95 . The results revealed a high degree of reliability in carrying out first and second measurements.

Kappa score was done for the condylar position and shapes to determine the agreement between two observers. The measure of agreement for the condylar position, Kappa

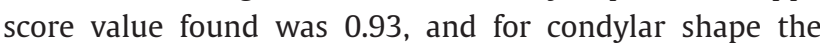


Kappa score value found was 0.84. A good agreement was demonstrated between the two observers in this study. The interpretation of Kappa score value was rated according to the Altman. ${ }^{24}$

\section{Results}

The study sample consisted of 22 patients among which 9 males and 13 females with class III malocclusion at their late adolescent period (mean age $=15 \pm 1.4$ years) were recruited in the study. A total of 7 Malay and 15 Chinese patients were included as shown in ( - Table 4$)$. No dropout was reported in the study.

There were no significant differences observed in condylar measurements (length, height, and width) and the RGF thickness. The condylar volume was increased after treatment, but no statistically significant differences ( $p=0.903$ right side and $p=0.062$ on left side) were observed on both sides.

Table 4 Demographic characteristics

\begin{tabular}{|l|l|}
\hline \multirow{2}{*}{ Variables } & Frequency (\%) \\
\cline { 2 - 2 } & ASSD ( $\boldsymbol{n}=\mathbf{2 2})$ \\
\hline Age & $15( \pm 1.4)$ \\
\hline Mean (SD) & $9(40.9)$ \\
\hline Mex & $13(59.09)$ \\
\hline Female & $7(31.81)$ \\
\hline Ethnic group & $15(68.18)$ \\
\hline Malay &
\end{tabular}

Abbreviations: ASSD, active skeletonized sutural distractor; SD, standard deviation.
Among spatial measurements, statistically significant differences were observed in posterior, anterior, and superior joint spaces on both right and left sides. The anterior joint space was increased significantly by $0.8 \mathrm{~mm}$ on both right and left side. It was followed by a significant decrease in superior and posterior joint spaces of approximately 0.2 and $0.5 \mathrm{~mm}$, respectively, post-ASSD treatment as shown in ( - Table 5 ).

The $90.9 \%$ of the subjects on right side revealed more anteriorly placed condyles, and less concentrically placed condyles, that is $9.09 \%$ with no condyles positioned posteriorly before treatment, whereas the position of the condyle presented more concentrically placed condyles $90.9 \%$ and less posteriorly placed condyles $9.09 \%$ with no condyles positioned anteriorly after treatment. The position of the condyles before treatment on the left side revealed more anteriorly placed condyles $81.8 \%, 4.54 \%$ of subjects revealed posteriorly placed condyles, and $13.63 \%$ had anteriorly placed condyles. Whereas the condylar position after treatment on the left side showed $95.45 \%$ concentrically placed condyles, $4.54 \%$ anteriorly placed condyles, but no posteriorly placed condyles were observed as shown in (-Table 6).

Regarding condylar morphology, the condylar shape before treatment on the right side revealed $36.36 \%$ convex condyles, 50\% rounded, and $13.63 \%$ angulated, whereas the condylar shape on right side presented $9.09 \%$ convex condyles, $31.81 \%$ rounded and $9.09 \%$ angulated condyles with no significant differences observed between the two groups after treatment. The left side of the condyles showed $27.27 \%$ convex condyles, $68.18 \%$ rounded and $4.54 \%$ angulated before treatment. The condylar shape on the left side presented 50\% convex condyles, $27.27 \%$ rounded and $22.72 \%$ angulated condyles with no significant differences between the two groups after treatment as shown in (-Table 7).

Table 5 Comparison of temporomandibular joint morphology between pre- and post-treatment measurements

\begin{tabular}{|c|c|c|c|c|c|c|c|c|}
\hline \multirow{3}{*}{$\begin{array}{l}\text { Dependent } \\
\text { variables } \\
(\mathrm{mm})\end{array}$} & \multirow{2}{*}{\multicolumn{2}{|c|}{$\begin{array}{c}\text { Right } \\
n=22 \\
\text { Median (IQR) }\end{array}$}} & \multirow{2}{*}{\multicolumn{2}{|c|}{$\begin{array}{c}\text { Left } \\
n=22 \\
\text { Median (IQR) }\end{array}$}} & \multirow{2}{*}{\multicolumn{2}{|c|}{ Z-Statistic ${ }^{\mathrm{a}}$}} & \multirow{2}{*}{\multicolumn{2}{|c|}{ p-Value ${ }^{a}$}} \\
\hline & & & & & & & & \\
\hline & Pre & Post & Pre & Post & Right & Left & Right & Left \\
\hline Condylar length & $7.9(0.40)$ & $8.0(0.43)$ & $7.75(0.30)$ & $7.9(0.23)$ & -1.653 & -1.567 & -0.098 & 0.117 \\
\hline Condylar width & $15.7(2.28)$ & $15.85(3.33)$ & $15.7(2.55)$ & $16(2.38)$ & -0.374 & -1.687 & 0.709 & 0.092 \\
\hline Condylar height & $18(1.65)$ & $18(1.60)$ & $18.35(1.75)$ & $18.4(1.93)$ & -1.701 & -1.107 & 0.089 & 0.268 \\
\hline RGF thickness & $1(0.10)$ & $0.9(0.05)$ & $1(0.10)$ & $0.9(0.40)$ & -1.310 & -1.003 & 0.190 & 0.316 \\
\hline $\begin{array}{l}\text { Anterior joint } \\
\text { space }\end{array}$ & $2(0.35)$ & $2.8(0.3)$ & $2.05(0.43)$ & $2.85(0.30)$ & -4.028 & -4.117 & $0.000^{\mathrm{a}}$ & $0.000^{\mathrm{a}}$ \\
\hline $\begin{array}{l}\text { Posterior joint } \\
\text { space }\end{array}$ & $2.3(0.6)$ & $2.1(0.43)$ & $2.3(0.35)$ & $2.2(0.33)$ & -3.873 & -3.843 & $0.000^{\mathrm{a}}$ & $0.000^{\mathrm{a}}$ \\
\hline $\begin{array}{l}\text { Superior joint } \\
\text { space }\end{array}$ & $1.9(0.4)$ & $1.7(0.43)$ & $2.05(0.70)$ & $2(0.40)$ & -4.062 & -2.821 & $0.000^{\mathrm{a}}$ & $0.005^{\mathrm{a}}$ \\
\hline $\begin{array}{l}\text { Condylar volume } \\
\left(\mathrm{mm}^{3}\right)\end{array}$ & $\begin{array}{l}1,077.73 \\
(357.21)\end{array}$ & $\begin{array}{l}1,094.36 \\
(456.59)\end{array}$ & $\begin{array}{l}1,021.53 \\
(379.61)\end{array}$ & $\begin{array}{l}1,206.87 \\
(513.48)\end{array}$ & -0.122 & -1.867 & 0.903 & 0.062 \\
\hline
\end{tabular}

Abbreviations: IQR, interquartile range; RGF, roof of glenoid fossa, $p=$ significant value $(p<0.05)$.

aWilcoxon paired signed rank test, ${ }^{*}=$ statistically significant, condylar volume in $\mathrm{mm}^{3}$.

Note: Linear measurement in $\mathrm{mm}$. 
Table 6 Comparison of condylar position between pre and post-treatment group

\begin{tabular}{|c|c|c|c|c|c|c|}
\hline \multirow{4}{*}{$\begin{array}{l}\text { Condylar } \\
\text { Position }\end{array}$} & \multicolumn{4}{|c|}{ * Statistically significant } & \multicolumn{2}{|c|}{$p$-Value } \\
\hline & \multicolumn{2}{|c|}{ Right } & \multicolumn{2}{|c|}{ Left } & \multirow[t]{3}{*}{ Right } & \multirow[t]{3}{*}{ Left } \\
\hline & Pre & Post & Pre & Post & & \\
\hline & $n(\%)$ & $n(\%)$ & $n(\%)$ & $n(\%)$ & & \\
\hline Anterior & 20 (90.9) & 0 & $18(81.8)$ & $1(4.54)$ & & \\
\hline Posterior & 0 & $2(9.09)$ & $1(4.54)$ & 0 & & \\
\hline Concentric & $2(9.09)$ & $20(90.9)$ & $3(13.63)$ & $21(95.45)$ & $0.000^{\mathrm{a}}$ & $0.000^{\mathrm{a}}$ \\
\hline Total (\%) & $22(100)$ & $22(100)$ & $22(100)$ & $22(100)$ & & \\
\hline Chi-square (df) & $14.727(1)$ & $14.727(1)$ & $23.545(1)$ & $18.182(1)$ & & \\
\hline
\end{tabular}

Abbreviations: df, degree of freedom; TM], temporomandibular joint.

*Statistically significant.

Table 7 Comparison of condylar shape between pre and post-treatment group

\begin{tabular}{|c|c|c|c|c|c|c|}
\hline \multirow{4}{*}{$\begin{array}{l}\text { Condylar } \\
\text { Shape }\end{array}$} & \multicolumn{4}{|c|}{ TMJ sides } & \multicolumn{2}{|r|}{$p$-Value } \\
\hline & \multicolumn{2}{|l|}{ Right } & \multicolumn{2}{|l|}{ Left } & \multirow[t]{3}{*}{ Right } & \multirow[t]{3}{*}{ Left } \\
\hline & Pre & Post & Pre & Post & & \\
\hline & $n(\%)$ & $n(\%)$ & $n(\%)$ & $n(\%)$ & & \\
\hline Convex & $8(36.36)$ & $13(59.09$ & $6(27.27)$ & $11(50)$ & & \\
\hline Round & $11(50)$ & $7(31.81)$ & $15(68.18)$ & $6(27.27)$ & & \\
\hline Angulated & $3(13.63)$ & $2(9.09)$ & $1(4.54)$ & $5(22.72)$ & $0.108^{a}$ & $0.244^{\mathrm{a}}$ \\
\hline Total (\%) & $22(100)$ & $22(100)$ & $22(100)$ & $22(100)$ & & \\
\hline Chi-square (df) & $4.452(2)$ & $6.636(2)$ & $2.818(2)$ & $2.818(2)$ & & \\
\hline
\end{tabular}

Abbreviations: df, degree of freedom; TMJ, temporomandibular joint.

*Statistically significant

\section{Discussion}

The characteristic of the mandibular condyle differs among various age groups and individuals. Morphologic variations may occur due to simple developmental changes as well as condylar remodeling to aid in developmental alterations, trauma, malocclusion, and other developmental deformities and disorders. A clear understanding of the TMJ anatomy and its morphology is required so that an abnormal condition can be determined when there is a difference in the normal variant. Our study focuses on various shapes and sizes of the mandibular condyle in healthy joints on both sides. The mandibular condyle changes significantly both in size and shape. ${ }^{25}$

Timing of the treatment for class III malocclusion that produces optimum results has always been a matter of extensive research over the past few years. Baccetti et $\mathrm{al}^{26}$ suggested skeletal age was ideally considered for older children rather than chronological age to obtain the desired results. The assessment of skeletal age was usually performed by using hand-wrist radiographs, but cervical maturation indicators have gained more popularity in recent years. Hence, cervical maturation indicators have been preferred over hand wrist radiographs for late adolescent age group patients. ${ }^{12}$

Some previous studies revealed the comparison of class III malocclusion among the younger and older age subjects with 8 to 13 years. The group I consisted of 47 subjects with rapid palatal expansion and group II comprised 13 subjects with labiolingual appliances. A maxillary protraction of $2 \mathrm{~mm}$ was achieved with palatal expansion and did not find any significant differences between the age groups. ${ }^{27}$

\section{Maxillary Advancement}

Rapid maxillary expansion (RME) appliances used in the treatment of class III malocclusion corrects transverse maxillary deficiency and malocclusion of anteroposterior origin. RME in combination with facemask therapy is often used for transversally underdeveloped maxilla, which requires greater amount of expansion. ${ }^{28}$ Its effect on the mandible includes an increase in width and spatial rotation giving rise to a significant increase in the distance of interglenoid fossa and condylar displacement leading to changes in the condylar position, which is considered as high potential for remodeling at the mean age of $11.4 \pm 1.5$ years, ${ }^{29}$ the results of this study are similar to our study findings.

It was suggested by Lin et $\mathrm{a}^{30}$ that, concerning the maxillary transverse dimension, the bone-anchored palatal expander increases the skeletal level than did the hyrax palatal expansion device at late adolescent age, in comparison with ASSD greater palatal expansion has been achieved in patients with late adolescent age who had limited growth potential. Several studies have utilized different appliances such as Facemask, Twin block appliance, and Jasper jumper to correct class III malocclusion, and the treatment duration 
ranged from 12 to 24 months, ${ }^{31-33}$ in comparison to ASSD which utilizes 6 months to correct class III malocclusion. In our study, an optimum force was applied on each side (500 g per side) to correct the skeletal discrepancy and protract the maxilla to obtain the desired result. However, no signs and symptoms of TMD have been reported.

A similar result has been reported in a study done by Lee et $\mathrm{al}^{33}$ in which a Delaire face mask (DFM) was used for maxillary protraction to treat skeletal class III malocclusion with maxillary deficiency and anterior crossbite. Patients were instructed to wear a facemask for 16 hours daily with $450 \mathrm{~g}$ protraction force for approximately 13 months. As a result, the condyle moves upward and backward giving rise to clockwise rotation of the mandible. In comparison, our study showed similar results by using ASSD for 6 months and the duration was less than half when compared with the use of facemask, and no clinical signs and symptoms of TMJ have been reported.

\section{Alternate Rapid Maxillary Expansion and Constriction}

Alternate rapid maxillary expansion and constriction (Alt-RAMEC) develops new bone by stretching the craniofacial sutures mechanically. The craniofacial sutures are osteogenic tissues connecting the opposing bones of the skull. The biological response to mechanical traction by the appliance includes the opening of the sutures, variation in the fiber bundle orientation, and increase in osteoblastic activity leading to deposition of formation a new bone on both surfaces of the sutural bones. RME aids in sutural expansion osteogenesis, whereas Alt-RAMEC aids in rapid maxillary protraction osteogenesis with alternate expansion and constriction. Histologically, these two treatment methods involve the separation of circummaxillary sutures of the palatal origin with concomitant osteogenic activity. ${ }^{27}$ The optimum orthopedic and functional occlusal forces associated with rapid maxillary expansion devices play a significant role in condylar responses. Thus RME/Alt-RAMEC treatment increases width of the maxilla and perimeter of the dental arch. ${ }^{34}$

Treatment protocol developed by Liou and $\mathrm{Tsai}^{35}$ in the Alt-RAMEC group consists of Alt-RAMEC 9 weeks, followed by 3 months and 3 weeks of maxillary protraction. The total treatment period was 6 months. Starting from the first day after the bonding, the expander was expanded and constricted to $1 \mathrm{~mm}$ daily for a week. The patients were reviewed every 3 weeks for replacing or adjusting the intraoral protraction springs. The significant anterior displacement of the maxilla and rotation of the mandible posteriorly and inferiorly was observed in both the groups. But maxillae and nasal bones were displaced more anteriorly in Alt-RAMEC group. The overall advancement of the maxilla in the Alt-RAMEC group was $5.8 \pm 2.3 \mathrm{~mm}$, and in the RME group was $2.6 \pm$ $1.5 \mathrm{~mm} .{ }^{36}$ Kaya et al used another method to increase the skeletal effect of maxillary protraction in which face mask was combined with miniplates placed on the lateral wall of the nasal maxillae, followed by 8-week Alt-RAMEC protocol. The authors demonstrate that retraction of mandibular incisors was observed without the movement of upper incisor teeth and maxillary advancement of $2 \mathrm{~mm}$ was noted over 9.9 months, and a counter-clockwise rotation of the maxillae occurred to 0.8 degrees. In comparison, this study has shown the desired expansion of 6 to $8 \mathrm{~mm}$ with an anticlockwise rotation of the maxilla by using Alt-RAMEC protocol in combination with temporary anchorage devices (TAD) and class III elastics.

\section{Class III Elastics and Mandibular Position}

Ngan and Moon $^{37}$ demonstrate that camouflage class III treatment in nongrowing patients often require MEAW with extractions in the lower arch and class III elastics. The elastics were used from mandibular teeth to retract the mandible and control the deep bite. The MEAW technique has many loops with second-order bends to control the vertical movements of the posterior teeth. It controls the movement of individual teeth and transmits the force produced by class III elastics. The entire mandibular arch can be postured back and uprighted by MEAW technique and intermaxillary arch elastics. This combined effect induces a clockwise rotation of the mandible, thus reducing the mandibular prognathism.

Al-Mozany et al $^{38}$ suggested that the combined use of AltRAMEC protocol with TADs in conjunction with class III heavy elastics produces a skeletal effect. The sample comprised 14 patients with the retrognathic maxilla. All patients were treated by using a mini-implant supported RME device, which was activated by Alt-RAMEC protocol for 9 weeks. The results were obtained within 8.5 weeks of the activation of Alt-RAMEC protocol. Protraction of the maxilla was done significantly while bringing the mandible to the backward position, and lower facial height was increased significantly leading to improvement in jaw relationship. In our study, the response of the condyle to ASSD treatment for 6 months gives rise to the rotation of the condyle in a clockwise direction leading to the displacement of the mandible in a superior and posterior direction.

\section{Morphological Changes of Temporomandibular Joint in Response to Active Skeletonized Sutural Distractor Treatment}

Comparison between pre and post-treatment variable measurements indicates that there were no statistically significant differences observed in condylar length, width, and height measurements. The roof of the glenoid fossa and condylar volume also showed no significant differences except the joint space measurements which showed statistically significant differences among anterior, posterior, and superior joint spaces. Anterior joint space was significantly increased after treatment, followed by a decrease in superior and posterior joint space giving rise to an upward and backward movement of the condyles on both sides. In our study, most of the condyles were in anterior position before ASSD treatment, indicating the class III malocclusion and later the condyles were in concentric position after the treatment with ASSD. A previous study was in agreement with our study results in which the authors demonstrated, the anterior joint space was increased followed by a decrease in posterior joint space in response to treatment and the condyle moved posteriorly with respect to vertical plane. ${ }^{39} \mathrm{El}$ and $\mathrm{Ciger}^{40}$ used two types of face mask to compare the condylar position. 
Thirty-four patients were treated with protraction face mask therapy were divided into two groups: 18 were treated with the DFM and 16 with the Grummons face mask (GFM). The observation periods were 8.5 months for the DFM group and 10 months for the GFM group. The authors reported that the discrepancy between centric relation and maximum intercuspation was less in the DFM group when compared with the GFM group. However, more compressive movement of the condyles through the glenoid fossa was noted in the DFM group, and no signs and symptoms of TMJ were observed.

The condylar shape changes were observed with the use of ASSD for 6 months. Before treatment, the more rounded shape of the condyles was noted on both right and left sides. After the treatment with ASSD, more convex shapes of the condyles were observed on both sides. Adaptive mechanism of mandibular condyles may cause bone remodeling with changes in the condylar structure leading to variation in the shape of the condyles. ${ }^{41} \mathrm{Few}$ studies have reported condylar remodeling in class III patients treated with orthognathic surgery. ${ }^{42,43}$ Peltola et al ${ }^{44}$ have reported flattened mandibular condyles in $9 \%$ of 625 treated subjects.

Merigue et al. ${ }^{45}$ evaluated the TMJ between class I and class II division 1 malocclusion subjects. The sample was divided into two groups: Group 1 had 26 class I subjects and group II had class II division 1 subjects. The authors reported convex shape (57.7\%), round (34.61\%), and angulated (7.69\%) were found in group 1; Group 2 revealed convex shape (75\%), round (20.83\%), and angulated (4.17\%), with no significant intergroup differences. Rawlani ${ }^{46}$ has reported $67 \%$ convex shapes, $29.2 \%$ flat, and $3.8 \%$ were rounded condyles. Similarly, Karlo et $\mathrm{al}^{47}$ have found rounded condylar shapes in growing children with the mean age of 7 years. The authors reported that the shape of the condyle was rounded initially, which later turns to an oval shape as the age advances.

In terms of the age of the subjects, they were selected in the late adolescent stage of growth to limit the amount of relapse after treatment. The unique characteristics of this study include the study sample was collected from 18 secondary schools of Kota Bharu in Kelantan by screening 13,500 students. It was considered a huge number in comparison, to similar studies which collected the sample size from the outpatient clinic only, not by screening the target population. However, our study has some limitations such as the follow-up period was 6 months, which is relatively short. The complete bony changes to take place a minimum duration of 10 to 12 months is required. Articular disc assessment was not done in this study. As it requires MRI assessment, which is expensive.

We did not have a sufficient budget to carry out the assessment. The future recommendations for this research include, the long-term follow-up of at least 10 to 12 months duration is required to determine whether the changes occurred in joint spaces and the position of the condyles are temporary or there are any chances of relapse. Further, prospective longitudinal studies with a large sample size should be conducted to determine the response of ASSD treatment on TMJ and its adjacent structures.

\section{Conclusion}

We can conclude with the treatment of ASSD with the onset of 6 months:

- The condyle-fossa relationship in class III malocclusion patients did not show any significant variation in condylar height, width, and length between right and left sides after treatment.

- The RGF and condylar volume also did not show any significant change. The significant changes were observed in joint spaces. The SJS and PJS were decreased after ASSD treatment. The AJS was increased significantly after treatment which gave rise to a corresponding change in the condylar position.

- The condylar position changes significantly in response to treatment with ASSD, most of the condyles were in concentric position after the treatment.

- Condylar shape changes in response to treatment with ASSD. Most of the condyles were rounded shape before treatment later changes to convex shape after the treatment.

\section{Funding}

This study was funded by Research University Individual (RUI) Grant (no: 1001/PPSG/8012216 USM RUI, RM94,880.00) of the Universiti Sains Malaysia.

\section{Conflict of Interest}

None declared.

\section{Acknowledgment}

The authors would like to thank Orthodontic Unit, School of Dental Sciences at University Sains Malaysia, Health campus, Kelantan-Malaysia.

\section{References}

1 Elfseyie M, Hassan M, Mohammed N, Al-Jaf A. Prevalence of malocclusion and occlusal traits of Malay adults (18-23 years) in Shah Alam, Malaysia. J Dent 2020:81-86

2 Soh J, Sandham A, Chan YH. Occlusal status in Asian male adults: prevalence and ethnic variation. Angle Orthod 2005;75(5):814-820

3 Sami al Jahmi RH, Roselinda AY, Prevalence and comparison of craniofacial morphology of class I, class II and class III malocclusions in kota bharu population. Thesis: Universiti Sains Malaysia; 2019

4 Behbehani F, Artun J, Al-Jame B, Kerosuo H. Prevalence and severity of malocclusion in adolescent Kuwaitis. Med Princ Pract 2005;14(6):390-395

5 El-Mangoury NH, Mostafa YA. Epidemiologic panorama of dental occlusion. Angle Orthod 1990;60(3):207-214

6 Gelgör IE, Karaman AI, Ercan E. Prevalence of malocclusion among adolescents in central anatolia. Eur J Dent 2007;1(3):125-131

7 Perillo L, Masucci C, Ferro F, Apicella D, Baccetti T. Prevalence of orthodontic treatment need in southern Italian schoolchildren. Eur J Orthod 2010;32(1):49-53

8 Sidlauskas A, Lopatiene K. The prevalence of malocclusion among 7-15-year-old Lithuanian schoolchildren. Medicina (Kaunas) 2009;45(2):147-152

9 Otuyemi OD, Abidoye RO. Malocclusion in 12-year-old suburban and rural Nigerian children. Community Dent Health $1993 ; 10(4): 375-380$ 
10 Rwakatema DS, Nganga PM, Kemoli AM. Prevalence of malocclusion among 12-15-year-olds in Moshi, Tanzania, using Bjork's criteria. East Afr Med J 2006;83(7):372-379

11 Hardy D. Prevalence of angle class III malocclusion: a systematic review and meta-analysis. Open J Epidemiol 2012;02:75-82

12 Zere E, Chaudhari PK, Sharan J, Dhingra K, Tiwari N. Developing Class III malocclusions: challenges and solutions. Clin Cosmet Investig Dent 2018;10:99-116

13 Azamian Z, Shirban F. Treatment options for class III malocclusion in growing patients with emphasis on maxillary protraction. Scientifica (Cairo) 2016;2016:8105163

14 Steinberg B, Padwa BL, Boyne P, Kaban L. State of the art in oral and maxillofacial surgery: treatment of maxillary hypoplasia and anterior palatal and alveolar clefts. Cleft Palate Craniofac J 1999;36(4):283-291

15 Ahn Y-S, Kim S-G, Baik S-M, et al. Comparative study between resorbable and nonresorbable plates in orthognathic surgery. J Oral Maxillofac Surg 2010;68(2):287-292

16 Al-Rawi NH, Uthman AT, Sodeify SM. Spatial analysis of mandibular condyles in patients with temporomandibular disorders and normal controls using cone beam computed tomography. Eur J Dent 2017;11(1):99-105

17 Krisjane Z, Urtane I, Krumina G, Zepa K. Three-dimensional evaluation of TMJ parameters in Class II and Class III patients. Stomatologija 2009;11(1):32-36

18 Katsavrias EG. Morphology of the temporomandibular joint in subjects with Class II Division 2 malocclusions. Am J Orthod Dentofacial Orthop 2006;129(4):470-478

19 Katayama K, Yamaguchi T, Sugiura M, Haga S, Maki K. Evaluation of mandibular volume using cone-beam computed tomography and correlation with cephalometric values. Angle Orthod 2014;84(2):337-342

20 The 2007 recommendations of the international commission on radiological protection. ICRP publication 103. Ann ICRP 2007;37(2-4):1-332

21 Al-koshab M, Nambiar P, John J. Assessment of condyle and glenoid fossa morphology using CBCT in South-East Asians. PLoS One 2015;10(3):e0121682

22 Pullinger AG, Solberg WK, Hollender L, Guichet D. Tomographic analysis of mandibular condyle position in diagnostic subgroups of temporomandibular disorders. J Prosthet Dent 1986;55(6):723-729

23 Kinzinger G, Kober C, Diedrich P. Topography and morphology of the mandibular condyle during fixed functional orthopedic treatment -a magnetic resonance imaging study. J Orofac Orthop 2007;68(2):124-147

24 Altman DG, Practical Statistics for Medical Research. CRC Press; 1990

25 Hegde S, Praveen B, Shetty S. Morphological and radiological variations of mandibular condyles in health and diseases: a systematic review. J Dent 2013;3(1):154

26 Baccetti T, Franchi L, McNamara JA Jr. The Cervical Vertebral Maturation (CVM) Method for the Assessment of Optimal Treatment Timing in Dentofacial Orthopedics. Elsevier; 2005 119-129

27 Al-Mozany S. Treatment of Class III malocclusions using temporary anchorage devices (TADs) the Alt RAMEC protocol and inermaxillary Class III elastics in the growing patient. Available at: https://ses.library.usyd.edu.au/bitstream/ handle/2123/7925/Treatment\%20of\%20Class\%20III\%20malocclusions\%20using\%20Temporary\%20Anchorage.pdf?sequence $=4 \&$ isAllowed=y. Accessed 2011

28 Ozzeybek Can FS, Turkkahraman H. Effects of rapid maxillary expansion and facemask therapy on the soft tissue profiles of class III patients at different growth stages. Eur J Dent 2019;13(2):143-149

29 Ghoussoub MS, Rifai K, Garcia R, Sleilaty G. Effect of rapid maxillary expansion on glenoid fossa and condyle-fossa relationship in growing patients (MEGP): study protocol for a controlled clinical trial. J Int Soc Prev Community Dent 2018;8(2):130-136

30 Lin L, Ahn H-W, Kim S-J, Moon S-C, Kim S-H, Nelson G. Tooth-borne vs bone-borne rapid maxillary expanders in late adolescence. Angle Orthod 2015;85(2):253-262

31 Kurt H, Alioğlu C, Karayazgan B, Tuncer N, Kılıçoğlu $\mathrm{H}$. The effects of two methods of Class III malocclusion treatment on temporomandibular disorders. Eur J Orthod 2011;33(6):636-641

32 Masucci C, Franchi L, Giuntini V, Defraia E. Short-term effects of a modified Alt-RAMEC protocol for early treatment of Class III malocclusion: a controlled study. Orthod Craniofac Res 2014;17(4):259-269

33 Lee $\mathrm{H}$, Son W-S, Kwak C, et al. Three-dimensional changes in the temporomandibular joint after maxillary protraction in children with skeletal Class III malocclusion. J Oral Sci 2016;58(4):501-508

34 Uzuner FD, Odabasi H, Acar S, Tortop T, Darendeliler N. Evaluation of the effects of modified bonded rapid maxillary expansion on occlusal force distribution: a pilot study. Eur J Dent 2016;10(1):103-108

35 Liou EJ, Tsai WC. A new protocol for maxillary protraction in cleft patients: repetitive weekly protocol of alternate rapid maxillary expansions and constrictions: Cleft Palate Craniofac J 2005;42(2):121-127

36 Kaya D, Kocadereli I, Kan B, Tasar F. Effects of facemask treatment anchored with miniplates after alternate rapid maxillary expansions and constrictions; a pilot study. Angle Orthod 2011;81(4):639-646

37 Ngan P, Moon W. Evolution of Class III treatment in orthodontics. Am J Orthod Dentofacial Orthop 2015;148(1):22-36

38 Al-Mozany SA, Dalci O, Almuzian M, Gonzalez C, Tarraf NE, Ali Darendeliler M. A novel method for treatment of Class III malocclusion in growing patients. Prog Orthod 2017;18(1):40

39 Alhammadi MS, Fayed MS, Labib A. Three-dimensional assessment of temporomandibular joints in skeletal Class I, Class II, and Class III malocclusions: cone beam computed tomography analysis. J World Fed Orthod 2016;5(3):80-86

$40 \mathrm{El} \mathrm{H}$, Ciger S. Effects of 2 types of facemasks on condylar position. Am J Orthod Dentofacial Orthop 2010;137(6):801-808

41 Vandeput A-S, Verhelst P-J, Jacobs R, Shaheen E, Swennen G, Politis C. Condylar changes after orthognathic surgery for class III dentofacial deformity: a systematic review. Int J Oral Maxillofac Surg 2019;48(2):193-202

42 Ha MH, Kim YI, Park SB, Kim SS, Son WS. Cone-beam computed tomographic evaluation osteotomy and rigid fixation. Korean J Orthod 2013;43(6):263-270

43 Park SB, Yang YM, Kim YI, Cho BH, Jung YH, Hwang DS. Effect of bimaxillary surgery on adaptive condylar head remodeling: metric analysis and image interpretation using cone-beam computed tomography volume superimposition. J Oral Maxillofac Surg 2012;70(8):1951-1959

44 Peltola JS, Nyström M, Könönen M, Wolf J. Radiographic structural findings in the mandibular condyles of young individuals receiving orthodontic treatment. Acta Odontol Scand 1995;53(2):85-91

45 Merigue LF. Conti ACdCF, Oltramari-Navarro PVP, Navarro RdL, Almeida MRd. Tomographic evaluation of the temporomandibular joint in malocclusion subjects. condylar morphology and position. Braz Oral Res 2016;30(1)

46 Rawlani S, Bhowate R, Kashikar S, Khubchandani M, Rawlani S, Chandak R. Morphological evaluation of temporo-mandibular joint in indian population. Braz Dent Sci 2018;21(1):44-53

47 Karlo CA, Stolzmann P, Habernig S, Müller L, Saurenmann T, Kellenberger CJ. Size, shape and age-related changes of the mandibular condyle during childhood. Eur Radiol 2010;20(10):2512-2517 